\title{
Dissimilarity-Based Classification of Anatomical Tree Structures
}

\author{
Lauge Sørensen ${ }^{1}$, Pechin Lo $^{1}$, Asger Dirksen ${ }^{2}$, Jens Petersen$^{1}$, and Marleen de \\ Bruijne ${ }^{1,3}$ \\ 1 The Image Group, Department of Computer Science, University of Copenhagen, \\ Denmark, lauges@diku.dk \\ 2 Department of Respiratory Medicine, Gentofte University Hospital, Denmark \\ 3 Biomedical Imaging Group Rotterdam, Departments of Radiology \& Medical \\ Informatics, Erasmus MC, The Netherlands
}

\begin{abstract}
A novel method for classification of abnormality in anatomical tree structures is presented. A tree is classified based on direct comparisons with other trees in a dissimilarity-based classification scheme. The pair-wise dissimilarity measure between two trees is based on a linear assignment between the branch feature vectors representing those trees. Hereby, localized information in the branches is collectively used in classification and variations in feature values across the tree are taken into account. An approximate anatomical correspondence between matched branches can be achieved by including anatomical features in the branch feature vectors. The proposed approach is applied to classify airway trees in computed tomography images of subjects with and without chronic obstructive pulmonary disease (COPD). Using the wall area percentage (WA\%), a common measure of airway abnormality in COPD, as well as anatomical features to characterize each branch, an area under the receiver operating characteristic curve of 0.912 is achieved. This is significantly better than computing the average WA\%.
\end{abstract}

Keywords: dissimilarity representation, linear assignment, tree classification, tree dissimilarity measure

\section{Introduction}

We consider the task of classifying abnormality in anatomical tree structures in medical images. Examples of such structures and applications are: quantification of airway tree abnormalities in, e.g., chronic obstructive pulmonary disease (COPD) [1-3] or asthma [4], quantification of arterial stenosis in the circle of Willis in the brain [5], and quantification of retinopathy of prematurity in retinal vessels [6]. The goal is to relate the global state of the anatomical tree to the disease status based on local information in the branches of the tree. This goal is often achieved by considering only parts of the tree and computing an average feature across the branches in those parts [1-6].

Abnormalities may be localized in different parts of the tree, and the underlying normal structure of the branches often varies across the tree. The value 
range of the feature used to capture the local information will therefore often be different for different parts of the tree, and the discriminative ability of a feature averaged across several branches may be limited. There may also be a functional difference between a tree containing one severely abnormal branch compared to a tree with several mildly abnormal branches, which can, however, give rise to the same average feature value.

In this paper, we propose to learn from data the relationship between the collective set of branches representing a tree and the global state of that tree. An anatomical tree is considered as a set of branches each characterized by one or more features, and a global label, the disease status of the subject, is associated with the entire tree. Classification is based on direct comparisons between trees, or sets of branch feature vectors, using a dissimilarity-based classification scheme [7]. The feature values extracted in individual branches are hereby collectively taken into account and related to the global state of the tree. A globally optimal one-to-one matching, according to a linear assignment [8], of the branches in two trees is used as anatomical tree dissimilarity measure on which the classification is based. More information is used compared to using an average feature since individual branches are compared, taking localized information into account which is combined into an overall tree dissimilarity measure.

Dissimilarity-based classification has previously proven useful in medical imaging, e.g., for detection of tuberculosis in chest radiographs [9] and for classification of emphysematous tissue in computed tomography (CT) [10]. A similar approach to the one proposed in this paper has previously been successfully applied to classify subjects using texture information in patches of lung tissue from CT images [11]. In the current paper, we consider tree structures. We experiment with different levels of "rigidness" by matching the branches solely based on an abnormality feature and by matching the branches using both abnormality and anatomical features. The latter approach ensures an approximate anatomical correspondence between matched branches. This is in contrast to tree proximity measures based on tree matching $[12,13]$, which are more computationally demanding.

The dissimilarity-based classification scheme is applied to detect COPD in volumetric pulmonary CT images using automatically segmented airway tree branches. COPD is a major public health problem that is projected to become the third leading cause of death worldwide by 2020 [14]. Pulmonary function tests (PFTs), which are the current gold standard for diagnosing and monitoring COPD [14], are insensitive to early stages of the disease and lack reproducibility, and there is therefore a general interest in finding good image-based markers for COPD. In this paper, the focus is on airway disease, one of the main components of COPD, that is mainly characterized by a narrowing of the smaller airways leading to limitations of the airflow to and from the alveoli. This narrowing is caused by inflammation and fibrosis, resulting in thicker airway walls, as well as mucus, blocking the passage of air in the lumen.

The proposed approach is evaluated and compared to computing the average wall area percentage (WA\%) across the branches in the tree. This is a commonly 
used CT-based marker of airway disease in the clinical literature that, despite its simplicity, have shown a significant correlation with PFTs [1-3].

\section{Classification in Tree Dissimilarity Space}

An anatomical tree $X=\left\{\mathbf{x}_{i}\right\}_{m}$ can be represented by a set of $m$ branch feature vectors $\mathbf{x}_{i}$, each feature describing the branch shape or appearance and/or the anatomical position of a branch. A label $Y$ is associated with the entire tree. The task is to classify an unseen tree $X$ based on a labeled training set of $n$ trees $\left\{\left(X_{i}, Y_{i}\right)\right\}_{n}$. Probabilistic classification outputs are considered as these can be directly interpreted as a probability of disease.

\subsection{Dissimilarity Representation}

A dissimilarity representation is used to classify the trees [7]. From the matrix of pair-wise tree dissimilarities $D=\left[d\left(X_{i}, X_{j}\right)\right]_{n \times n}$ computed from the training set $T=\left\{X_{i}\right\}_{n}$, there are different ways to derive a feature vector space where traditional vector space methods can be applied. In this work, we consider tree dissimilarity measures $d(\cdot, \cdot)$ that do not produce positive definite matrices, and the dissimilarity space approach, which does not require fulfillment of this condition, is therefore used [7]. An anatomical tree dissimilarity space is constructed of dimension equal to the size of the training set. Each feature vector describing a tree $X$ consists of the dissimilarities $d\left(X, X_{i}\right)$ to each of the trees in the training set, i.e., $D(X, T)=\left[d\left(X, X_{1}\right), d\left(X, X_{2}\right), \ldots, d\left(X, X_{n}\right)\right]$.

\subsection{Dissimilarity Measure}

The anatomical tree dissimilarity measure $d(\cdot, \cdot)$ is the crucial component in this construction, and it is in this part of the proposed approach that a set of branch feature vectors are collectively considered as representing a tree. The dissimilarity measure based on a linear assignment between sets of sub-objects proposed in [11] is used for this purpose. The dissimilarity between two anatomical trees, or sets of branches, $X_{1}=\left\{\mathbf{x}_{1 i}\right\}_{m}$ and $X_{2}=\left\{\mathbf{x}_{2 j}\right\}_{n}$ where $\mathbf{x}_{1 i}$ is the $i$ th branch feature vector in $X_{1}$, is expressed as the minimum linear sum assignment between the two sets where the cost of assigning $\mathbf{x}_{1 i}$ to $\mathbf{x}_{2 j}$ is the dissimilarity between those two branch feature vectors according to a branch dissimilarity measure $\Delta\left(\mathbf{x}_{1 i}, \mathbf{x}_{2 j}\right)$. This can be seen as assigning all the branches in the smallest tree to the branches in the other tree in a way such that the two trees are as similar as possible while only allowing one-to-one matchings. The linear assignment problem can be formulated in terms of a bipartite graph. However, it is important to note that this construction is only used in order to compute the linear assignment between the sets of branches and that no structure is imposed on the trees.

Let $G=\left(X_{1} \cup X_{2}, E\right)$ be a weighted undirected bipartite graph with node sets $X_{1}$ and $X_{2}$ where $\left|X_{1}\right|=\left|X_{2}\right|=n$, edge set $E=\left\{\left\{\mathbf{x}_{1 i}, \mathbf{x}_{2 j}\right\}: i, j=1, \ldots, n\right\}$, 
and with a weight $\Delta\left(\mathbf{x}_{1 i}, \mathbf{x}_{2 j}\right)$ associated with each edge $\left\{\mathbf{x}_{1 i}, \mathbf{x}_{2 j}\right\} \in E$. A subset $M$ of $E$ is called a perfect matching, or assignment, if every node of $\mathrm{G}$ is incident with exactly one edge in $M$. The perfect matching with minimum weight $M^{*}$ is given by

$$
M^{*}=\underset{M}{\operatorname{argmin}} \sum_{\left\{\mathbf{x}_{1 i}, \mathbf{x}_{2 j}\right\} \in M} \Delta\left(\mathbf{x}_{1 i}, \mathbf{x}_{2 j}\right): M \text { is a perfect matching. }
$$

This problem can be solved efficiently using the Hungarian algorithm [8]. The resulting anatomical tree dissimilarity measure is thus

$$
d_{l a}\left(X_{1}, X_{2}\right)=\sum_{\left\{\mathbf{x}_{1 i}, \mathbf{x}_{2 j}\right\} \in M^{*}} \Delta\left(\mathbf{x}_{1 i}, \mathbf{x}_{2 j}\right)
$$

where $M^{*}$ is obtained via (1).

Segmented anatomical trees are likely to contain differing number of branches, i.e., $\left|X_{1}\right| \neq\left|X_{2}\right|$, and the above formulation does not account for this. However, a problem with different number of branches in the two trees can be turned into a problem with the same number of branches by adding "dummy nodes" to the graph [8]. The resulting problem is now in the form of (1) and can again be solved using the Hungarian algorithm. Differences in tree sizes, in terms of number of branches, are accounted for by dividing by the number of branches in the smallest tree, in order not to favor smaller trees

$$
d_{l a n}\left(X_{1}, X_{2}\right)=\frac{d_{l a}\left(X_{1}, X_{2}\right)}{\min \left(\left|X_{1}\right|,\left|X_{2}\right|\right)} .
$$

A branch dissimilarity measure $\Delta(\cdot, \cdot)$ is needed in order to compute the anatomical tree dissimilarity measure $d_{l a n}(\cdot, \cdot)$. In this work, branches are represented by a $d$-dimensional feature vector $\mathbf{x}_{i}=\left[x_{i 1}, \ldots, x_{i d}\right]^{T}$, and the branch dissimilarity is computed as the Euclidean distance in the branch feature space $\Delta\left(\mathbf{x}_{i}, \mathbf{x}_{j}\right)=\left\|\mathbf{x}_{i}-\mathbf{x}_{j}\right\|_{2}$

\subsection{Classification}

Trees are classified by mapping into the anatomical tree dissimilarity space using $D(\cdot, T)$ followed by classification using the $k$ nearest neighbor $(k \mathrm{NN})$ classifier posterior probability estimate with the training set trees as prototypes

$$
p(Y \mid D(X, T))=\frac{k_{Y}(D(X, T))}{k}
$$

where $k_{Y}(D(X, T))$ is the number of nearest neighbors of $X$, in the dissimilarity space, belonging to class $Y$ out of a total of $k$ nearest neighbors. The Euclidean distance between the feature vector representation of the trees of $X$ and $X_{i}$ in the dissimilarity space, $\left\|D(X, T)-D\left(X_{i}, T\right)\right\|_{2}$, is used as distance in the $k \mathrm{NN}$ classifier. $k$ is fixed according to $k=\sqrt{n}$ where $n$ is the number of prototypes in the $k \mathrm{NN}$ classifier [15]. 


\section{Extraction of Airway Tree Branch Feature Vectors}

In order to extract the branch feature vectors representing each airway tree, the whole airway tree is segmented in the CT image into lumen and outer wall using the algorithm described in [16]. Figure 1(a) shows an example of a segmented airway tree using this algorithm. The segmentation is subdivided into branches and branch centerlines are extracted using the algorithm described in [17]. Figure 1 (b) shows a coloring of the identified branches using this algorithm. Branch generations are obtained by assigning generation 0 to the trachea and incrementing generation number by one when propagating the generation number from a parent centerline to its child centerlines. All the steps taken are fully automatic, and the reader is referred to [16,17] for further details.

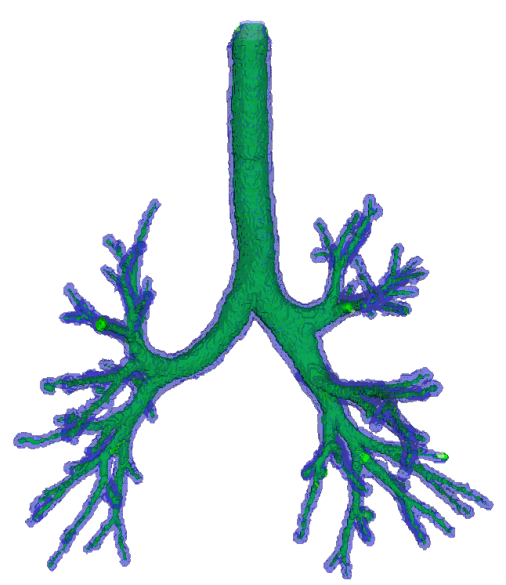

(a)

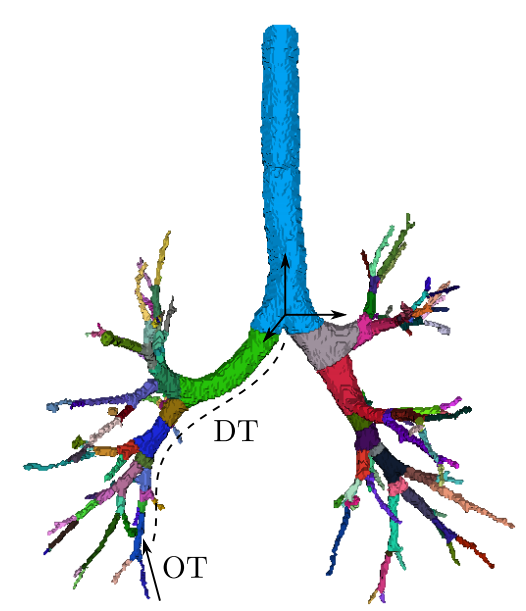

(b)

Fig. 1. (a) Airway segmentation with the inner airway wall shown in green and the outer airway wall shown in transparent blue. (b) Branches found in the airway segmentation shown in (a) overlayed with anatomical features.

Each branch is represented by a 5 -dimensional feature vector $\mathbf{x}_{i}$ comprising one measure known to be related to COPD as well as four anatomical features roughly capturing the location and orientation of the branch in the airway tree. The features are the following:

\section{COPD feature}

- wall area percentage (WA\%). The number of voxels in the branch wall relative to the total amount of voxels in a branch, i.e., the number of voxels in both the wall and the lumen. WA\% is computed from the segmented airway tree (lumen and outer wall). This is the most commonly used measure of 
airway disease in CT [1-3,18]. It is also used in asthma [4]. WA\% is computed from cross-sectional slices in the mentioned references whereas it is computed from volumetric segmentations of the branches in this paper. The reader is referred to [16] for details.

Anatomical features The anatomical features are computed from the centerline representation of the tree.

- distance to the trachea (DT). The distance from the root of the branch to the carina along the centerlines connecting that branch to the trachea. DT is scaled w.r.t. the subject size by dividing by the height of the subject. This feature can be seen as a continuous version of the branch generation, and is less affected by errors in the segmentation and branch detection.

- orientation w.r.t. the trachea (OT). The orientation of the branch in a global coordinate system based on the trachea, carina, and main bronchi. This is expressed as a three-dimensional vector.

The global coordinate system as well as the anatomical features for a specific branch are illustrated in Fig. 1(b). Each branch feature is standardized to zero mean and unit variance.

\section{Experiments}

\subsection{Data}

The data consists of 296 low-dose volumetric CT images from 296 participants of the Danish Lung Cancer Screening Trial [19] with the following scan parameters: tube voltage $120 \mathrm{kV}$, exposure $40 \mathrm{mAs}$, slice thickness $1 \mathrm{~mm}$, and in-plane resolution ranging from 0.72 to $0.78 \mathrm{~mm}$. 144 images are from subjects without COPD and 152 images are from subjects diagnosed with mild to very severe COPD according to spirometry [14].

\subsection{Evaluation}

The proposed anatomical tree classification approach is evaluated using leaveone-out estimation on the CT data, and the area under the receiver operating characteristic (ROC) curve (AUC) is computed on the soft outputs of the classifier of Eqn. (4). The anatomical tree dissimilarity space considered in each leave-out trial is of dimension equal to the size of the training set, i.e., 295dimensional. The proposed approach is compared to average WA\%, and the analysis is performed for different individual generations in the airway tree in the range 3 to 7 and for several ranges from 3-4 to 3-7. Similar generations were considered in $[1,2,20]$.

Different feature representations of the branches are considered, i.e., using WA\% only as well as using WA\% jointly with anatomical features in order to enforce an approximate anatomical correspondence in the matching. 


\subsection{Results}

The results are reported in Table 1 . The best overall performing measure is based on matching branches in generations 3 to 7 using WA\% as well as anatomical features. This is significantly better than using the average WA\% in all considered generation ranges $\left(p<10^{-4}\right)$ according to DeLong, DeLong, and Clarke-Pearson's test [21]. It is also significantly better than matching branches in generations 3 to 7 using WA\% without implicit correspondence by anatomical features $(p=0.001)$, but not significantly better than using anatomical features only $(p=0.613)$. ROC-curves for the underlined AUCs in Table 1 are shown in Fig. 2(a).

Table 1. AUCs for COPD diagnosis for the proposed approach (top part) and for average WA\% (bottom part). The best performing measure in each column is marked in bold-face, the best performing measure for each approach both for using an individual generation and a range of generations is underlined, and the overall best performing measure is marked in italics.

\begin{tabular}{|c|c|c|c|c|c|c|c|c|c|}
\hline \multirow[t]{2}{*}{ approach } & \multicolumn{5}{|c|}{ generation } & \multicolumn{4}{|c|}{ generations range } \\
\hline & 3 & 4 & 5 & 6 & 7 & $3-4$ & $3-5$ & $3-6$ & $3-7$ \\
\hline \multicolumn{10}{|l|}{$d_{l a n}$} \\
\hline WA $\%$ & 0.792 & 0.856 & 0.813 & 0.883 & 0.884 & 0.840 & 0.865 & 0.878 & 0.892 \\
\hline $\mathrm{WA} \%+\mathrm{DT}+\mathrm{OT}$ & 0.828 & 0.867 & 0.864 & $\underline{0.897}$ & 0.880 & 0.868 & 0.881 & 0.904 & $\underline{0.912}$ \\
\hline $\mathrm{DT}+\mathrm{OT}$ & 0.678 & 0.659 & 0.739 & 0.891 & 0.871 & 0.706 & 0.857 & 0.893 & 0.908 \\
\hline \multicolumn{10}{|l|}{ average feature } \\
\hline $\mathrm{WA} \%$ & 0.811 & $\underline{0.858}$ & 0.838 & 0.762 & 0.704 & $\underline{0.849}$ & 0.845 & 0.818 & 0.788 \\
\hline
\end{tabular}

The effect on the matching in Eqn.(1) of including anatomical branch features, in terms of how often a branch from one generation is matched to other generations, is inspected in Table 2. More branches from the same generations are matched when including anatomical features compared to using WA\% only, i.e., a larger percentage of the matches is concentrated in the diagonal of the matrix for $\mathrm{WA} \%+\mathrm{DT}+\mathrm{OT}$.

\section{Discussion}

Average WA\% performed best at generation 4. Considering more generations, in isolation or as a range, deteriorated the performance, see Table 1. WA\% on average grows as a function of generation, as seen in Fig. 2(b). This is due to anatomy, but also likely due to the resolution limit of the CT image being reached leading to size overestimation because of partial volume effects. This 
Table 2. Distribution of how often branches of different generations in the range 3 to 7 are matched in the whole data set when considering WA\% and when considering WA\% together with anatomy, respectively. The percentages of a total of 43660 matches are reported, and the total number of branches in generations $3-7$ are as follows: 2467, 4927, 8633, 10004, 7366. Note that these matrices are symmetric.

\begin{tabular}{|c|c|c|c|c|c|c|c|}
\hline & \multicolumn{3}{|c|}{ WA $\%$} & \multicolumn{4}{|c|}{$\mathrm{WA} \%+\mathrm{DT}+\mathrm{OT}$} \\
\hline & 34 & 5 & 6 & 34 & 5 & 6 & 7 \\
\hline 3 & 1.6 & & & 5.1 & & & \\
\hline 4 & 1.83 .1 & & & 1.86 .6 & & & \\
\hline 5 & 1.73 .9 & 6.9 & & 0.84 .41 & 10.2 & & \\
\hline 6 & 1.33 .5 & 7.81 & 10.0 & 0.32 .1 & 7.81 & 11.7 & \\
\hline 7 & 0.92 .5 & 5.7 & 7.45 .4 & 0.10 .8 & 3.6 & & 8.1 \\
\hline
\end{tabular}

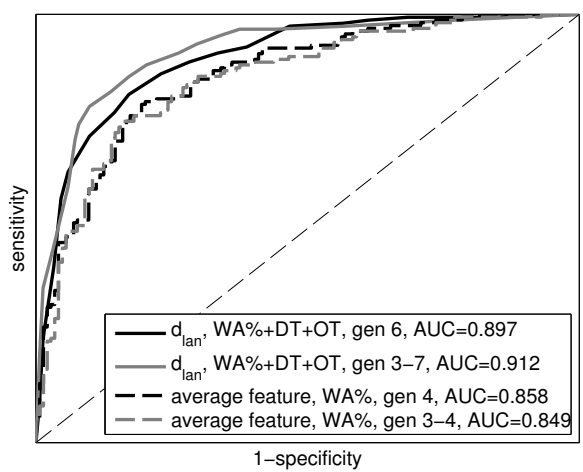

(a)

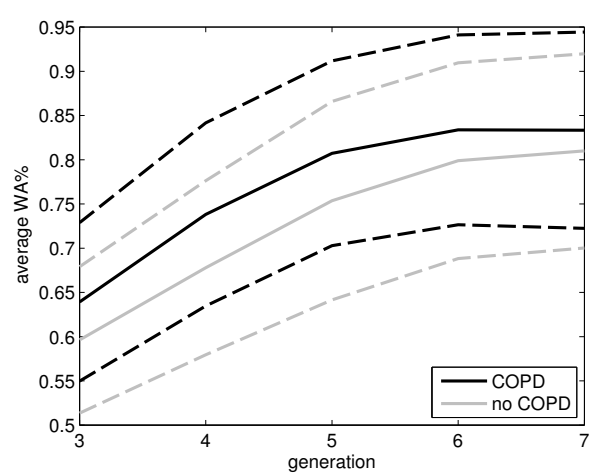

(b)

Fig. 2. (a) ROC-curves using the best performing generation and generations range for each approach corresponding to the underlined AUCs in Table 1. (b) WA\% as a function of generation for the two groups. Solid line is the mean and dashed lines are the mean \pm the standard deviation. 
Table 3. AUCs for COPD diagnosis on the pruned trees for the proposed approach (top part) and for using an average COPD feature (bottom part). The best performing measure in each column is marked in bold-face, the best performing measure for each approach both for using an individual generation and a range of generations is underlined, and the overall best performing measure is marked in italics. Results are not reported for generation 6 and 7 since some pruned trees did not contain branches in these generations.

\begin{tabular}{|c|c|c|c|c|c|c|c|c|c|}
\hline \multirow[t]{2}{*}{ approach } & \multicolumn{5}{|c|}{ generation } & \multicolumn{4}{|c|}{ generations range } \\
\hline & 3 & 4 & 5 & 6 & 7 & $3-4$ & $3-5$ & $3-6$ & $3-7$ \\
\hline \multicolumn{10}{|l|}{$d_{l a n}$} \\
\hline WA $\%$ & 0.792 & 0.856 & 0.813 & - & - & 0.840 & 0.848 & 0.861 & 0.859 \\
\hline $\mathrm{WA} \%+\mathrm{DT}+\mathrm{OT}$ & 0.828 & $\underline{0.867}$ & 0.864 & - & - & 0.868 & 0.885 & 0.886 & $\underline{0.887}$ \\
\hline $\mathrm{DT}+\mathrm{OT}$ & 0.678 & 0.659 & 0.739 & - & - & 0.705 & 0.759 & 0.750 & 0.747 \\
\hline \multicolumn{10}{|l|}{ average feature } \\
\hline WA $\%$ & 0.811 & $\underline{0.858}$ & 0.814 & - & - & 0.849 & 0.861 & $\underline{0.862}$ & $\underline{0.862}$ \\
\hline
\end{tabular}

variation in feature value across the tree may explain why average WA\% deteriorates for larger generations, i.e., the discriminative information is lost. The proposed approach naturally deals with this phenomenon by matching individual branches and can therefore incorporate branches from larger generations while preserving discriminative information. This may explain the significantly better performance.

The segmentation algorithm generally finds more branches in airway trees from the no COPD group compared to the COPD group in the data set. This skew in the number of branches may bias the results since partial matchings are allowed in Eqn. (3). This could explain why using anatomical branch features alone performs well, see Table 1 . We inspect this phenomenon by pruning the smallest branches in all the trees in the data set to the size of the smallest tree. The leave-one-out estimation is then repeated for the pruned trees, and the results are shown in Table 3. As seen, it is still possible to achieve high AUCs, and the proposed approach achieves the best AUC of 0.887 which is still significantly better than average WA\% for all the considered generation ranges $\left(p<10^{-4}\right)$. Moreover, the performance of using only anatomical features has become significantly worse than average WA\% in all cases $\left(p<10^{-4}\right)$.

The computational complexity of the proposed anatomical tree dissimilarity measure $d_{\text {lan }}\left(X_{1}, X_{2}\right)$ is bounded by the complexity of the algorithm used to compute the linear assignment. Using the Hungarian algorithm this is $\mathcal{O}\left(m^{4}\right)$ [8] where $m$ is the number of branches in the larger tree, i.e., $m=\max \left(\left|X_{1}\right|,\left|X_{2}\right|\right)$. This is in contrast to NP-complete tree proximity measures that take the tree topology into account, such as $[12,13]$. A test tree $X$ is classified by mapping into the anatomical tree dissimilarity space which has complexity $\mathcal{O}\left(\mathrm{nm}^{4}\right)$ where $n$ is the size of the training set, i.e., $n=|T|$ and $m$ in this case is the number of 
branches in the largest three among all training set trees as well as the test tree, i.e., $m=\max \left(|X|,\left|X_{1}\right|,\left|X_{2}\right|, \ldots,\left|X_{n}\right|\right), X_{i} \in T$. This is followed by classification in the dissimilarity space using a trained classifier.

The relative contribution of airway disease and emphysema, the other main component of COPD, to COPD varies [1] and may in fact be independent [22]. It would therefore be interesting to combine the current approach with measures of emphysema.

\section{Conclusions}

We propose a novel method for classification of abnormality in anatomical tree structures that is based on dissimilarities computed directly between anatomical trees as represented by sets of branch feature vectors. This is an alternative to a common approach in the clinical literature of averaging one branch feature or measuring a small number of specific locations. A $k \mathrm{NN}$ classifier in the dissimilarity space obtained using a tree dissimilarity measure based on a linear assignment between the branches in the trees using WA\% together with anatomical features, achieved an AUC of 0.912 on a COPD classification task in volumetric pulmonary CT. This was significantly better than the AUC of using the average WA\%.

Acknowledgements. This work is partly funded by the Netherlands Organisation for Scientific Research (NWO), and AstraZeneca, Sweden.

\section{References}

1. Hasegawa, M., Nasuhara, Y., Onodera, Y., Makita, H., Nagai, K., Fuke, S., Ito, Y., Betsuyaku, T., Nishimura, M.: Airflow limitation and airway dimensions in chronic obstructive pulmonary disease. Am J Respir Crit Care Med 173(12) (2006) 13091315

2. Achenbach, T., Weinheimer, O., Biedermann, A., Schmitt, S., Freudenstein, D., Goutham, E., Kunz, R.P., Buhl, R., Dueber, C., Heussel, C.P.: MDCT assessment of airway wall thickness in COPD patients using a new method: correlations with pulmonary function tests. Eur Radiol 18(12) (2008) 2731-2738

3. Washko, G.R., Dransfield, M.T., Estépar, R.S.J., Diaz, A., Matsuoka, S., Yamashiro, T., Hatabu, H., Silverman, E.K., Bailey, W.C., Reilly, J.J.: Airway wall attenuation: a biomarker of airway disease in subjects with COPD. J Appl Physiol 107(1) (2009) 185-191

4. Kasahara, K., Shiba, K., Ozawa, T., Okuda, K., Adachi, M.: Correlation between the bronchial subepithelial layer and whole airway wall thickness in patients with asthma. Thorax 57(3) (2002) 242-246

5. Roher, A.E., Esh, C., Kokjohn, T.A., Kalback, W., Luehrs, D.C., Seward, J.D., Sue, L.I., Beach, T.G.: Circle of willis atherosclerosis is a risk factor for sporadic alzheimer's disease. Arterioscler Thromb Vasc Biol 23(11) (2003) 2055-2062

6. Heneghan, C., Flynn, J., O'Keefe, M., Cahill, M.: Characterization of changes in blood vessel width and tortuosity in retinopathy of prematurity using image analysis. Med Image Anal 6(4) (2002) 407-429 
7. Pekalska, E., Duin, R.P.W.: The Dissimilarity Representation for Pattern Recognition: Foundations And Applications (Machine Perception and Artificial Intelligence). World Scientific Publishing Co., Inc. (2005)

8. Wolsey, L.A.: Integer Programming. Wiley-Interscience (1998)

9. Arzhaeva, Y., Hogeweg, L., de Jong, P.A., Viergever, M.A., van Ginneken, B.: Global and local multi-valued dissimilarity-based classification: Application to computer-aided detection of tuberculosis. In Yang, G.Z., Hawkes, D.J., Rueckert, D., Noble, J.A., Taylor, C.J., eds.: Medical Image Computing and Computer Assisted Intervention. Volume 5761 of Lecture Notes in Computer Science., Springer (2009) $724-731$

10. Sørensen, L., de Bruijne, M.: Dissimilarity representations in lung parenchyma classification. In Karssemeijer, N., Giger, M., eds.: Medical Imaging: ComputerAided Diagnosis. Volume 7260 of Proceedings of SPIE. (2009)

11. Sørensen, L., Loog, M., Lo, P., Ashraf, H., Dirksen, A., Duin, R.P.W., de Bruijne, M.: Image dissimilarity-based quantification of lung disease from CT. In Jiang, T., Navab, N., Pluim, J.P.W., Viergever, M.A., eds.: Medical Image Computing and Computer Assisted Intervention. Volume 6361 of Lecture Notes in Computer Science., Springer (2010) 37-44

12. Bille, P.: A survey on tree edit distance and related problems. Theor. Comput. Sci. 337 (2005) 217-239

13. Feragen, A., Lauze, F., Lo, P., de Bruijne, M., Nielsen, M.: Geometries on spaces of treelike shapes. In Kimmel, R., Klette, R., Sugimoto, A., eds.: Asian Conference on Computer Vision. Volume 6493 of Lecture Notes in Computer Science., Springer (2010) 160-173

14. Rabe, K.F., Hurd, S., Anzueto, A., Barnes, P.J., Buist, S.A., Calverley, P., Fukuchi, Y., Jenkins, C., Rodriguez-Roisin, R., van Weel, C., Zielinski, J.: Global strategy for the diagnosis, management, and prevention of chronic obstructive pulmonary disease: GOLD executive summary. Am. J. Respir. Crit. Care Med. 176(6) (2007) $532-555$

15. Kittler, J., Alkoot, F.M.: Moderating k-NN classifiers. Pattern Analysis and Applications 5(3) (2002) 326-332

16. Petersen, J., Nielsen, M., Lo, P., Saghir, Z., Dirksen, A., de Bruijne, M.: Optimal graph based segmentation using flow lines with application to airway wall segmentation. In Székely, G., Hahn, H., eds.: Information Processing in Medical Imaging. Lecture Notes in Computer Science, Springer (2011)

17. Lo, P., van Ginneken, B., Reinhardt, J., de Bruijne, M.: Extraction of airways from CT (EXACT'09). In Brown, M., de Bruijne, M., van Ginneken, B., Kiraly, A., Kuhnigk, J.M., Lorenz, C., McClelland, J., Mori, K., Reeves, A., Reinhardt, J., eds.: Proc. of The Second International Workshop on Pulmonary Image Analysis. (2009)

18. Nakano, Y., Wong, J.C., de Jong, P.A., Buzatu, L., Nagao, T., Coxson, H.O., Elliott, W.M., Hogg, J.C., Paré, P.D.: The prediction of small airway dimensions using computed tomography. Am J Respir Crit Care Med 171(2) (2005) 142-146

19. Pedersen, J.H., Ashraf, H., Dirksen, A., Bach, K., Hansen, H., Toennesen, P., Thorsen, H., Brodersen, J., Skov, B.G., Døssing, M., Mortensen, J., Richter, K., Clementsen, P., Seersholm, N.: The Danish randomized lung cancer CT screening trial-overall design and results of the prevalence round. J Thorac Oncol 4(5) (2009) 608-614

20. Hasegawa, M., Makita, H., Nasuhara, Y., Odajima, N., Nagai, K., Ito, Y., Betsuyaku, T., Nishimura, M.: Relationship between improved airflow limitation and 
changes in airway calibre induced by inhaled anticholinergic agents in COPD. Tho$\operatorname{rax}$ 64(4) (2009) 332-338

21. DeLong, E.R., DeLong, D.M., Clarke-Pearson, D.L.: Comparing the areas under two or more correlated receiver operating characteristic curves: a nonparametric approach. Biometrics 44(3) (1988) 837-845

22. Patel, B.D., Coxson, H.O., Pillai, S.G., Agustí, A.G.N., Calverley, P.M.A., Donner, C.F., Make, B.J., Müller, N.L., Rennard, S.I., Vestbo, J., Wouters, E.F.M., Hiorns, M.P., Nakano, Y., Camp, P.G., Fauerbach, P.V.N., Screaton, N.J., Campbell, E.J., Anderson, W.H., Paré, P.D., Levy, R.D., Lake, S.L., Silverman, E.K., Lomas, D.A., Network, I.C.G.: Airway wall thickening and emphysema show independent familial aggregation in chronic obstructive pulmonary disease. Am J Respir Crit Care Med 178(5) (2008) 500-505 